\title{
Sensitivities and Linear Stability Analysis Around a Double-Zero Eigenvalue
}

\author{
A. Luongo, ${ }^{*}$ A. Paolone, ${ }^{\dagger}$ and A. Di Egidio ${ }^{\dagger}$ \\ Università di L'Aquila, 67040 L'Aquila, Italy
}

\begin{abstract}
A general, multiparameter system admitting a double-zero eigenvalue at a critical equilibrium point is considered. A sensitivity analysis of the critical eigenvalues is performed to explore the neighborhood of the critical point in the parameter space. Because the coalescence of the eigenvalues implies that the Jacobian matrix is defective (or nilpotent), well-suited techniques of perturbation analysis must be employed to evaluate the eigenvalues and the eigenvector sensitivities. Different asymptotic methods are used, based on perturbations both of the eigenvalue problem and the characteristic equation. The analysis reveals the existence of a generic (nonsingular) case and of a nongeneric (singular) case. However, even in the generic case, a codimension-1 subspace exists in the parameter space on which a singularity occurs. By the use of the relevant asymptotic expansions, linear stability diagrams are builtup, and different bifurcation mechanisms (divergence-Hopf, double divergence, double divergence-Hopf, degenerate Hopf $\mathrm{f}$ are highlighted. The problem of finding a unique expression uniformly valid in the whole space is then addressed. It is found that a second-degree algebraic equa tion governs the behavior of the critical eigenvalues. It also permits elarification of the geometrical meaning of the unfolding parameters, which has been discussed in literature for the Takens-Bogdanova bifurcation. Finally, a meehanical system loaded by noneonservative forces and exhibiting a double-zero bifurcation is studied as an example.
\end{abstract}

\section{Nomenclature}

$\boldsymbol{A}=$ system Jacobian matrix where $\boldsymbol{x}$ is equal to 0

$\boldsymbol{A}_{0}=$ system Jacobian matrix where $\boldsymbol{x}$ is equal to $\boldsymbol{0}$ and $\boldsymbol{\theta}$ is equal to 0

$B$ = bifurcation locus for $I_{n}$

$C$ = critical locus of double zero eigenvalues

$\mathcal{D}=$ divergence boundary

$F \quad=$ vector field

$\mathcal{H}=$ Hopf boundary

$I_{k}=k$ th invariant of $\boldsymbol{A}$

$m$ = number of system parameters

$\mathcal{N}=$ nilpotent system locus

$n=$ dimension of $\boldsymbol{A}$

$S=$ singular codimension-1 subspace and first-order divergence boundary

$U \quad=$ matrix of right eigenvectors $u$

$\boldsymbol{u}_{k} \quad=$ proper right eigenvectors of $\boldsymbol{A}_{0}$ ( $k$ is greater than 2)

$\boldsymbol{u}_{1}=$ proper right eigenvector of $\boldsymbol{A}_{0}$ associated with $\lambda$ equal to 0

$\boldsymbol{u}_{2}=$ generalized right eigenvector of $\boldsymbol{A}_{0}$ associated with $\lambda$ equal to 0

$V$ = matrix of left eigenvectors $v$

$v_{k}=$ proper left eigenvectors of $\boldsymbol{A}_{0}$ ( $k$ is greater than 2)

$v_{1}=$ generalized left eigenvector of $\boldsymbol{A}_{0}$ associated with $\lambda$ equal to 0

$\boldsymbol{v}_{2}=$ proper left eigenvector of $\boldsymbol{A}_{0}$ associated with $\lambda$ equal to 0

$\boldsymbol{w}=$ right eigenvector of $\boldsymbol{A}$

$x=$ vector of the state variables

$\varepsilon \quad=$ perturbation parameter

$\lambda=$ eigenvalue of $A$

$\theta=$ control parameter vector $\{\alpha, \beta, \ldots\}$

\section{Introduction}

S ENSITIVITY analysisplays an importantrole in structuralmechanics. It permits description of the behavior of a system in which several parametersare varied around a given set of values with

'Professor, Dipartimento di Ingegneria delle Strutture, delle Acque e del Terreno; luongo@ ing .univaq, it.

${ }^{\dagger}$ Assistant Professor, Dipartimento di Ingegneria delle Strutture, delle Acque e del Terreno. a modest computationaleffort. Therefore, it is very useful in structural modification ${ }^{1}$ and system identification analysis. ${ }^{2}$ Many of the numerous studies on this subject have been devoted to the occurrence of multiple eigenvaluesin conservativesystems ${ }^{3-5}$ However. little attention has been paid to multiple eigenvalues in nonconservative systems, in which the system matrix becomes defective (or nilpotent, requiring the use of special techniques. ${ }^{6.7}$

Sensitivity analysis is also important to study bifurcation and stability in nonconservative systems. In linear problems, it makes it possible to explore the neighborhood of the critical manifold in the parameter space and to build up the linear stability diagram. In nonlinear analysis, it allows evaluation of the unfolding parameters that appear in the bifurcation equations, which are just proportional to the eigenvalue sensitivities, that is, to the derivatives of the eigenvalues with respect to the parameters. ${ }^{8}$

Among codimension- 2 bifurcations, the double-zero bifurcation is of particular interest. It calls for the analysis of a multiple eigenvalue for which the system matrix is defective. However, although this bifurcation, known as the Takens-Bogdanova bifurcation, has been widely discussed in the literature (for example, see Refs. 9 and 10) attention has been focused mainly on nonlinear behavior, and sensitivities of the critical eigenvalues have not been studied in depth. For example, it is stated in Refs. 11 and 12 that the mathematical unfolding parameters are quasi parallel to the tangent at the critical boundaries(in contrast to other types of bifurcationsin which they are exactly parallel), but this surprising result is neither elaborated nor justified.

A second question related to the double-zerobifurcation is the following. A codimension-2 bifurcation occurs in the parameter space at the intersection of two codimension-1 critical manifolds on each of which one eigenvalue has a zero real part. Thus, for example, a double Hopf bifurcation occurs at the intersection of two manifolds on which a simple Hopf bifurcation manifestsitself. In contrast, it is known that the Takens-Bogdanova bifurcation occurs at the intersection of a divergencemanifold and a single Hopf manifold. As a result of the interaction, the two Hopf imaginary eigenvalues collapse in the origin of the complex plane. However, it has not yet been clarified whether the divergenceHopfoccurrenceis exhaustiveor if other mechanisms leading to the double-zero bifurcation exist under particular conditions. In principle a double divergence or a degenerate simple Hopf bifurcationshould al so cause a double-zeroeigenvalue.

The aims of this paper are 1) to determine the conditions under which the Takens-Bogdanovabifurcation occurs and to investigate 
alternative bifurcation mechanisms, 2) to explore the neighborhood of the double-zeroeigenvalue to obtain analytical approximationsof both eigenvalues and eigenvectors, and 3) to clarify the question of the parallelism to the criticalboundariesof the unfolding parameters.

To reach these goals the following methodology is adopted: First, it is assumed that a critical point is known at which the system possesses a double-zero eigenvalue; second, its neighborhood is analyzed to determine the type of criticalboundariespassing through the point. Therefore, the procedure usually followed in the applications, which consists of evaluating the critical point at the intersection of known critical boundaries, is upward.

In Sec. II, the eigenvalue sensitivity analysis is carried out on the characteristic equation of the eigenvalue problem. In Sec. III, a different type of analysis is presented, based on a perturbation of both eigenvalues and eigenvectors. In Sec. IV, use is made of the preceding asymptotic expansions to build up the linear stability diagram. The analysis reveals the existence of a singularity that makes it impossible to use a unique expansion in the whole parameter space. The problem is overcome in Sec. V, where an equivalent second-degreecharacteristic equation is obtained that is able to capture all of the aspects of the problem. In Sec. VI, a sample problem is illustrated.

\section{Eigenvalue Sensitivity Analysis}

Let us consider a dynamic system $\dot{\boldsymbol{x}}=\boldsymbol{F}(\boldsymbol{x} ; \boldsymbol{\theta}), \boldsymbol{x} \in \mathfrak{R}^{n}$, depending on the parameter vector $\boldsymbol{\theta}=\{\alpha, \beta, \ldots\} \in \mathfrak{R}^{m}$. Let $\boldsymbol{x}=\mathbf{0}$ be an equilibrium point for any value of $\boldsymbol{\theta}$, that is, $\boldsymbol{F}(\mathbf{0}, \boldsymbol{\theta})=\mathbf{0} \forall \boldsymbol{\theta}$. The stability of the trivial equilibrium position is governed by the linear eigenvalue problem

$$
A w-\lambda w=0
$$

where $\boldsymbol{A}=\boldsymbol{A}(\boldsymbol{\theta}):=\boldsymbol{F}_{\boldsymbol{x}}(\mathbf{0}, \boldsymbol{\theta})$ is the Jacobian matrix at $\boldsymbol{x}=\mathbf{0}$, depending on $\boldsymbol{\theta}$. The characteristic polynomial of $\boldsymbol{A}$ reads

$$
\lambda^{n}+I_{1} \lambda^{n-1}+\cdots+I_{n-3} \lambda^{3}+I_{n-2} \lambda^{2}+I_{n-1} \lambda+I_{n}=0
$$

where $I_{k}=I_{k}(\boldsymbol{\theta})(k=1,2, \ldots, n)$ are the invariants of $\boldsymbol{A}$, also depending on $\boldsymbol{\theta}$. For Eq. (2) to admit the double root $\lambda=0$, then $I_{n}=0$ and $I_{n-1}=0$ must hold simultaneously for the same values of $\boldsymbol{\theta}$. The two equations describe two codimension- 1 manifolds, whose intersection is a codimension- 2 critical locus $C$ of doublezero eigenvalues. However, whereas $I_{n}=0$ is a simple divergence boundary, no eigenvalue properties are associated with $I_{n-1}=0$. Therefore, only one divergence boundary crosses the critical manifold $C$.

An exception occurs when $C$ contains a bifurcation locus $\mathcal{B}$ for $I_{n}$, at which multiple divergence manifests itself. This special case requires that all of the derivatives $I_{n, \theta}$ vanish at $\mathcal{B}$ and, therefore, represents a higher codimension bifurcation. However, it should be stressed that, to have a multiple divergence, it is not sufficient that $I_{n}$ bifurcate; in fact if $I_{n-1} \neq 0$ at $\mathcal{B}$, Eq. (2) admits only one zero eigenvalue. This circumstance occurs, for example, in mechanical systems having a stiffness matrix depending on two load parameters, at least one of which is of follower type. For a suitable choice of parameters, the stiffness matrix possesses two zero eigenvalues; however, the damping destroys this coalescence and the system admits only one zero root. On the other hand, if the damping is small, it is expected that a second root is close to zero, so that the system is nearly defective. To study sensitivities of a nearly defective system, the techniqueillustratedin Ref. 13 should be used, which is based on the assumption of the system as a perturbation of an ideal defective system; therefore, a double-zero eigenvalue must again be studied. Such an analysis, however, will be not developed in this paper.

A perturbativeanalysis of the characteristicequation(2) furnishes insight into the behavior of the critical eigenvalues. Let us assume that, at $\boldsymbol{\theta}=\mathbf{0}, I_{k}^{0}:=I_{k}(\mathbf{0})=0(k=n-1, n)$. When $\boldsymbol{\theta}$ is small, for example, $\boldsymbol{\theta}:=\varepsilon \boldsymbol{\theta}$ with $\varepsilon \ll 1$ and $\boldsymbol{\theta}=\mathcal{O}(1)$, it is $I_{n-1}=\varepsilon \hat{I}_{n-1}^{0}+$ $\mathcal{O}\left(\varepsilon^{2}\right)$ and $I_{n}=\varepsilon \hat{I}_{n}^{0}+\mathcal{O}\left(\varepsilon^{2}\right)$, with $\hat{I}_{k}^{0}=I_{k, \theta}^{0} \boldsymbol{\theta}(k=n-1, n)$, provided at least one parameter derivative is different from zero (generic case, in which the invariants do not bifurcate at $\boldsymbol{\theta}=\mathbf{0})$. By substituting $I_{n}$ and $I_{n-1}$ in Eq. (2), we have, by inspection, that $\lambda=\mathcal{O}\left(\varepsilon^{1 / 2}\right)$; therefore, by expanding $\lambda$ as $\lambda=\varepsilon^{1 / 2} \lambda_{1 / 2}+\varepsilon \lambda_{1}+\mathcal{O}\left(\varepsilon^{3 / 2}\right)$, we have the following perturbation equations that are drawn from Eq. (2):

$$
\begin{gathered}
\varepsilon: I_{n-2}^{0} \lambda_{\frac{1}{2}}^{2}+\hat{I}_{n}^{0}=0 \\
\varepsilon^{\frac{3}{2}}:\left(I_{n-3}^{0} \lambda_{\frac{1}{2}}^{2}+2 I_{n-2}^{0} \lambda_{1}+\hat{I}_{n-1}^{0}\right) \lambda_{\frac{1}{2}}=0
\end{gathered}
$$

By solving Eqs. (3), we have

$$
\begin{gathered}
\lambda_{\frac{1}{2}}= \pm \sqrt{-\frac{\hat{I}_{n}^{0}}{I_{n-2}^{0}}} \\
\lambda_{1}=-\frac{1}{2 I_{n-2}^{0}}\left(\hat{I}_{n-1}^{0}-I_{n-3}^{0} \frac{\hat{I}_{n}^{0}}{I_{n-2}^{0}}\right)
\end{gathered}
$$

from which an asymptotic expression for $\lambda$, corrected up to the $\varepsilon$ order, is obtained. However, it must be observed that this asymptotic solution holds only if $\lambda_{1 / 2} \neq 0$, that is, if $\hat{I}_{n}^{0} \neq 0$ [see Eq. (3b)]. Indeed, although the derivatives $I_{n, \theta}^{0}$ are not all zero by hypothesis, a singular case occurs if the perturbation is tangent to the manifold $I_{n}=0$ because $I_{n, \theta}^{0} \theta=0$. Therefore, the analysis also reveals a singularity in the generic case.

On the other hand, if $I_{n, \boldsymbol{\theta}}^{0} \equiv \mathbf{0}$, that is, if $\boldsymbol{\theta}=\mathbf{0}$ is a bifurcation point for $I_{n}$, then $I_{n}=\varepsilon^{2} \hat{\hat{I}}_{n}^{0}$, with $\hat{\hat{I}}_{n}^{0}=\frac{1}{2} I_{n, \boldsymbol{\theta} \theta} \boldsymbol{\theta}^{2}$. By Eq. (2) it follows that $\lambda=\mathcal{O}(\varepsilon)$. By letting $\lambda=\varepsilon \lambda_{1}+\mathcal{O}\left(\varepsilon^{2}\right)$, we have the lower-order perturbation equation

$$
\varepsilon^{2}: I_{n-2}^{0} \lambda_{1}^{2}+I_{n-1}^{0} \lambda_{1}+\hat{I}_{n}^{0}=0
$$

from which two values of $\lambda_{1}$ are drawn.

Equation (5) also makes it possible to analyze the singular perturbation in the generic case. By assuming $I_{n, \theta}^{0} \theta$ is small of order $\varepsilon$ and redefining $\hat{I}_{n}^{0}$ as $\hat{I}_{n}^{0}=I_{n, \boldsymbol{\theta}}^{0} \boldsymbol{\theta}+\frac{1}{2} I_{n, \boldsymbol{\theta} \theta}^{0} \boldsymbol{\theta}^{2}$, we have Eq. (5) describing the behavior of the critical eigenvalues around the singularity.

Although the preceding analysis reveals the essential aspects of the problem, it is not completely satisfactory. In fact, it requires knowledge of the four invariants of highest order of the matrix $\boldsymbol{A}$, whose evaluationis not easy for large matrices. On the other hand, it would be convenient to obtain the expressions of the sensitivities directly involving the coefficients of the matrix $\boldsymbol{A}$ and its perturbations. To this end, the analysis described in Ref. 6, which is developed on the eigenvalue problem (1) rather than on its characteristic equation (2), seems to be more suitable. Moreover, this technique allows determination of sensitivities of both eigenvalues and eigenvectors, the latter also being of interest. The relevant analysis is developed in the next section.

\section{Eigenpair Sensitivity Analysis}

Eigenvalue problem (1) is considered again. It is assumed that, when $\boldsymbol{\theta}=\mathbf{0}$, the matrix $\boldsymbol{A}_{0}:=\boldsymbol{A}(\mathbf{0})$ admits a double $\lambda=0$ (critical) eigenvalue, while the remaining (passive) eigenvalues are distinct, of order $\mathcal{O}(1)$, and have negative real parts. Let $\boldsymbol{u}_{1}$ be the unique eigenvector of $\boldsymbol{A}_{0}$ associated with $\lambda=0 ; \boldsymbol{A}_{0}$ is, therefore, defective (or nilpotent). To analyze the dependence of both criticaleigenvalues and eigenvectors on the parameters, a sensitivity analysis of the eigenpairs of $\boldsymbol{A}_{0}$ around $\boldsymbol{\theta}=\mathbf{0}$ must be performed.

Some of the algebraic properties of $\boldsymbol{A}_{0}$ are the following. Because $\boldsymbol{A}_{0}$ is defective, its eigenvectors do not span the whole space. To complete the base, generalized eigenvectors must be used. Two generalized right eigenvectors $\left\{\boldsymbol{u}_{1}, \boldsymbol{u}_{2}\right\}$ associated with the double $\lambda=0$ eigenvalue exist, the proper eigenvector $\boldsymbol{u}_{1}$ and the order-two eigenvector $\boldsymbol{u}_{2}$, that satisfy $\boldsymbol{A}_{0} \boldsymbol{u}_{1}=\mathbf{0}$ and $\boldsymbol{A}_{0} \boldsymbol{u}_{2}=\boldsymbol{u}_{1}$, respectively. Similarly, two generalized left eigenvectors $\left\{\boldsymbol{v}_{1}, \boldsymbol{v}_{2}\right\}$ associated with the double $\lambda=0$ exist, the proper eigenvector $v_{2}$ and the order-one eigenvector $\boldsymbol{v}_{1}$, that satisfy $\boldsymbol{A}_{0}^{T} \boldsymbol{v}_{2}=\mathbf{0}$ and $\boldsymbol{A}_{0}^{T} \boldsymbol{v}_{1}=\boldsymbol{v}_{2}$, respectively. The two sets of eigenvectors are biorthogonal, that is, $\boldsymbol{v}_{i}^{T} \boldsymbol{u}_{j}=\delta_{i j}$, if suitable normalizations are introduced. Because $\boldsymbol{v}_{2}^{T} \boldsymbol{u}_{2} \nRightarrow 0$, eigenvector $\boldsymbol{u}_{2}$ does not belong to the range of $\boldsymbol{A}_{0}$.

To analyze the dependence on $\boldsymbol{\theta}$ of the critical eigenvalues around $\boldsymbol{\theta}=\mathbf{0}$, curves passing through the origin of the parameter space are considered, with parametric equations $\boldsymbol{\theta}=\boldsymbol{\theta}(\varepsilon)$, where $\varepsilon$ is a 
parameter; along these curves $\boldsymbol{A}=\boldsymbol{A}[\boldsymbol{\theta}(\varepsilon)]$. By expanding in series the parametric equations as

$$
\boldsymbol{\theta}=\varepsilon \boldsymbol{\theta}_{1}+\varepsilon^{2} \boldsymbol{\theta}_{2}+\cdots
$$

we have the Jacobian matrix along the curves

$$
\boldsymbol{A}=\boldsymbol{A}_{0}+\varepsilon \boldsymbol{A}_{1}+\varepsilon^{2} \boldsymbol{A}_{2}+\cdots
$$

where

$$
A_{1}=A_{\theta}^{0} \theta_{1}, \quad A_{2}=A_{\theta}^{0} \theta_{2}+\frac{1}{2} A_{\theta \theta}^{0} \boldsymbol{\theta}_{1}^{2}
$$

are the first- and second-order perturbation matrices, respectively. By following Ref. 6 and according to Sec. II, we have the eigenpairs $(\lambda, w)$ that are expanded in series of fractional powers of $\varepsilon$ :

$$
\begin{gathered}
\lambda=\varepsilon^{\frac{1}{2}} \lambda_{\frac{1}{2}}+\varepsilon \lambda_{1}+\varepsilon^{\frac{3}{2}} \lambda_{\frac{3}{2}}+\varepsilon^{2} \lambda_{2}+\cdots \\
\boldsymbol{w}=\boldsymbol{w}_{0}+\varepsilon^{\frac{1}{2}} \boldsymbol{w}_{\frac{1}{2}}+\varepsilon \boldsymbol{w}_{1}+\varepsilon^{\frac{3}{2}} \boldsymbol{w}_{\frac{3}{2}}+\varepsilon^{2} \boldsymbol{w}_{2}+\cdots
\end{gathered}
$$

By substituting them in Eq. (1), and using Eq. (7), we obtain the following perturbation equations:

$$
\begin{gathered}
\varepsilon^{0}: \boldsymbol{A}_{0} \boldsymbol{w}_{0}=\mathbf{0} \\
\varepsilon^{\frac{1}{2}}: \boldsymbol{A}_{0} \boldsymbol{w}_{\frac{1}{2}}=\lambda_{\frac{1}{2}} \boldsymbol{w}_{0} \\
\varepsilon: \boldsymbol{A}_{0} \boldsymbol{w}_{1}=\lambda_{\frac{1}{2}} \boldsymbol{w}_{\frac{1}{2}}+\lambda_{1} \boldsymbol{w}_{0}-\boldsymbol{A}_{1} \boldsymbol{w}_{0} \\
\varepsilon^{\frac{3}{2}}: \boldsymbol{A}_{0} \boldsymbol{w}_{\frac{3}{2}}=\lambda_{\frac{1}{2}} \boldsymbol{w}_{1}+\lambda_{1} \boldsymbol{w}_{\frac{1}{2}}+\lambda_{\frac{3}{2}} \boldsymbol{w}_{0}-\boldsymbol{A}_{1} \boldsymbol{w}_{\frac{1}{2}} \\
\varepsilon^{2}: \boldsymbol{A}_{0} \boldsymbol{w}_{2}=\lambda_{\frac{1}{2}} \boldsymbol{w}_{\frac{3}{2}}+\lambda_{1} \boldsymbol{w}_{1}+\lambda_{\frac{3}{2}} \boldsymbol{w}_{\frac{1}{2}}+\lambda_{2} \boldsymbol{w}_{0}-\boldsymbol{A}_{1} \boldsymbol{w}_{1}-\boldsymbol{A}_{2} \boldsymbol{w}_{0}
\end{gathered}
$$

To avoid indeterminate quantities, Eqs. (10) must be equipped with suitable normalization conditions. By requiring $\boldsymbol{v}_{1}^{T} \boldsymbol{w}=1$, we have the conditions $\boldsymbol{v}_{1}^{T} \boldsymbol{w}_{0}=1$ and $\boldsymbol{v}_{1}^{T} \boldsymbol{w}_{j}=0\left(j=\frac{1}{2}, 1, \ldots\right)$ follow from Eq. (9b).

By solving the $\varepsilon^{0}$ - and $\varepsilon^{1 / 2}$-order perturbation equations, we obtain $\boldsymbol{w}_{0}=\boldsymbol{u}_{1}$ and $\boldsymbol{w}_{1 / 2}=\lambda_{1 / 2} \boldsymbol{u}_{2}$, with $\lambda_{1 / 2}$ stillundetermined. To solve the $\varepsilon$-order equation, the component of the known term external to the range of the singularoperator $A_{0}$ must be removed. By enforcing orthogonality to $\boldsymbol{v}_{2}$, we find $\lambda_{1 / 2}$ :

$$
\lambda_{\frac{1}{2}}= \pm\left(v_{2}^{T} A_{1} u_{1}\right)^{\frac{1}{2}}
$$

Equation (10c) can then be solved to furnish $\boldsymbol{w}_{1}=\hat{\boldsymbol{w}}_{1}+\lambda_{1} \boldsymbol{u}_{2}$, where $\hat{\boldsymbol{w}}_{1}$ is the (unique) solution to the problem

$$
\begin{gathered}
\boldsymbol{A}_{0} \hat{\boldsymbol{w}}_{1}=\lambda_{\frac{1}{2}}^{2} \boldsymbol{u}_{2}-\boldsymbol{A}_{1} \boldsymbol{u}_{1} \\
\boldsymbol{v}_{1}^{T} \hat{\boldsymbol{w}}_{1}=0
\end{gathered}
$$

From Eqs. (11) and (12) it follows that $\hat{\boldsymbol{w}}_{1}$ is a linearhomogeneous function of $\boldsymbol{\theta}_{1}$. Accounting for earlierresults, we have the solvability condition of the $\varepsilon^{3 / 2}$-order perturbation equation

$$
\lambda_{\frac{1}{2}}\left(2 \lambda_{1}-v_{2}^{T} A_{1} u_{2}+v_{2}^{T} \hat{w}_{1}\right)=0
$$

from which $\lambda_{1}$ is determined, provided $\lambda_{1 / 2} \neq 0$. By expressing $\hat{\boldsymbol{w}}_{1}$ in the base of the right eigenvectors, we find that $\boldsymbol{v}_{2}^{T} \hat{\boldsymbol{w}}_{1}=-\boldsymbol{v}_{1}^{T} \boldsymbol{A}_{1} \boldsymbol{u}_{1}$ (see the Appendix), so that

$$
\lambda_{1}=\frac{1}{2}\left(v_{2}^{T} A_{1} u_{2}+v_{1}^{T} A_{1} u_{1}\right)
$$

The procedure would possibly be continued at higher orders, although the two-term expansions

$$
\lambda= \pm \varepsilon^{\frac{1}{2}}\left(\boldsymbol{v}_{2}^{T} \boldsymbol{A}_{1} \boldsymbol{u}_{1}\right)^{\frac{1}{2}}+(\varepsilon / 2)\left(\boldsymbol{v}_{2}^{T} \boldsymbol{A}_{1} \boldsymbol{u}_{2}+\boldsymbol{v}_{1}^{T} \boldsymbol{A}_{1} \boldsymbol{u}_{1}\right)+\mathcal{O}\left(\varepsilon^{\frac{3}{2}}\right)
$$

for the eigenvalues and the three-term expansions

$$
\boldsymbol{w}=\boldsymbol{u}_{1}+\varepsilon^{\frac{1}{2}} \lambda_{\frac{1}{2}} \boldsymbol{u}_{2}+\varepsilon\left(\lambda_{1} \boldsymbol{u}_{2}+\hat{\boldsymbol{w}}_{1}\right)+\mathcal{O}\left(\varepsilon^{\frac{3}{2}}\right)
$$

for the eigenvectors are usually accurate. ${ }^{6}$ However, as observed in Sec. II, this asymptotic solution holds only if $\lambda_{1 / 2} \neq 0$, that is, only if the $m \times 1$ matrix

$$
L:=v_{2}^{T} A_{\theta} u_{1}=\left[v_{2}^{T} A_{\alpha} u_{1}, v_{2}^{T} A_{\beta} u_{1}, \ldots\right]
$$

has maximum rank, that is, if at least one coefficient of $\boldsymbol{L}$ is different from zero; otherwisea nongenericcase occurs. Moreover, even in the generic case, a singular codimension- 1 subspace $\mathcal{S}$ of the parameter space exists, on which $\lambda_{1 / 2}=\boldsymbol{L} \boldsymbol{\theta}_{1}=0$.

To analyze sensitivities of the critical eigenvalues 1) in the whole space in the nongeneric case and 2) in the subspace $\mathcal{S}$ in the generic case, the eigenpairs $(\lambda, w)$ should be expanded in series of integer powers of $\varepsilon$ (see Ref. 6 ). The same results are achieved here by using the perturbationscheme of Eqs. (10) and going up to the $\varepsilon^{2}$ order, although this procedure is less straightforward. When $\lambda_{1 / 2}=0$, Eq. (13) identically vanishes, so that $\lambda_{1}$ is still undetermined at $\varepsilon^{3 / 2}$ order. By solving Eqs. $\left(10_{4}\right)$, we find $\boldsymbol{w}_{3 / 2}=\mathbf{0}$, and the solvability conditions of the $\varepsilon^{2}$-order perturbation equation are

$$
\lambda_{1}^{2}+\xi \lambda_{1}+\kappa^{*}=0
$$

where

$$
\begin{gathered}
\xi:=-\left(v_{2}^{T} A_{1} u_{2}+v_{1}^{T} A_{1} u_{1}\right) \\
\kappa^{*}:=-\left(v_{2}^{T} A_{2} u_{1}+v_{2}^{T} A_{1} \hat{w}_{1}\right)
\end{gathered}
$$

and $\hat{\boldsymbol{w}}_{1}$ satisfies Eqs. (12) with $\lambda_{1 / 2}=0$. Therefore, $\lambda=\varepsilon \lambda_{1}+\mathcal{O}\left(\varepsilon^{2}\right)$, with $\lambda_{1}$ given by Eq. (18). Note from Eq. (19) that, differently from the nonsingular expansion Eq. (15), the critical eigenvalues now depend on the passive eigenvalues through $\hat{\boldsymbol{w}}_{1}$ (see the Appendix), although the two solutions are both corrected up to the $\varepsilon$ order.

Equations (13), (14), and (18) have the same structure as Eqs. (4a), (4b), and (5) obtained by the earlier analysis. However, their coefficients can be more easily evaluated and reveal properties that are hidden in the analysis of Sec. II. One of these has been observed; a second one is that the coefficient $\xi$ in Eqs. (19) is proportional to $\lambda_{1}$ in Eqs. (14). This circumstance does not appear in Eqs. (4b) and (5) because $\hat{I}_{n-1}^{0}$ has different expressions in the generic and the nongeneric cases.

\section{Stability Diagram}

The stability diagram can be built from the sensitivities of the critical eigenvalues. The generic case in which $\boldsymbol{L}$ has maximum rank is considered first.

Equation (15) is used to describe the eigenvalues in the whole space by ignoring the existence of a singularity (rough analysis). By the requirement that $\lambda_{1 / 2}=0$, the codimension-1(singular) subspace $\mathcal{S}$ is found, which divides the space into two half-spaces (see Fig. 1, where a bidimensional case is represented). At the leading order, the eigenvalues are real and opposite in one half-space and complex conjugate in the other half-space. By the requirement that $\lambda_{1}=0$, because $\lambda_{1}$ is linear and homogeneous in $\theta_{1}$, a new codimension- 1 half-subspace $\mathcal{H}$ is found that divides the subspace of the complex eigenvalues into a stable and an unstable domain. The subspace $\mathcal{S}$ is a first-order divergence boundary and the half-subspace $\mathcal{H}$ a first-order Hopf boundary.

From Fig. 1, no regions exist in which the two eigenvalues are real and positive, in spite of the occurrence of a double divergence at $\boldsymbol{\theta}=\mathbf{0}$. This result is incorrect, due to the presence of a singularity on $\mathcal{S}$. To understand better the mechanism causing the drawback, note

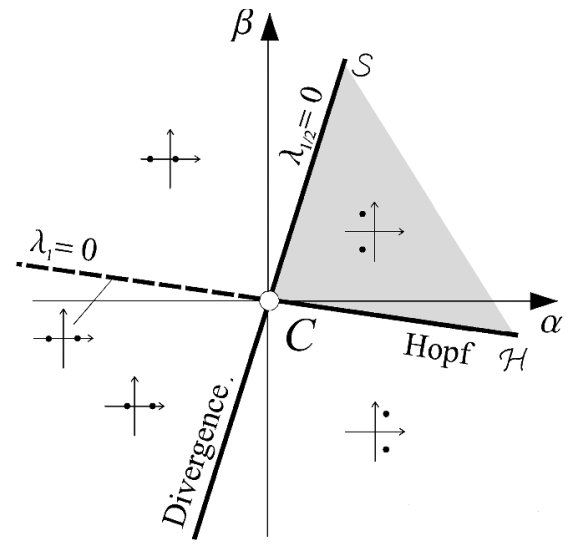

Fig. 1 Stability diagram in the generic case: rough analysis. 
that only the first-order part $\boldsymbol{\theta}_{1}$ of the parameters $\boldsymbol{\theta}$ appears at the $\varepsilon$ order of the $\lambda$ expansion. Therefore, the parameter space is spanned by straight lines originating from $\boldsymbol{\theta}=\mathbf{0}$. When these lines are close to $\mathcal{S}, \lambda_{1 / 2}$ is different from zero (generic case) but it is small, so that an ordering violation occurs. To remove the singularity and explore the neighborhood of $\mathcal{S}$, it is convenient to use curve lines tangent to $\mathcal{S}$ for which $\lambda_{1 / 2}=0$ exactly occurs (refined analysis). In this way $\lambda=\varepsilon \lambda_{1}$ holds, with $\lambda_{1}$ given by Eq. (18). Because $\boldsymbol{\theta}_{1}$ has already been determined, the coefficient $\xi$ in Eq. (18) is also known (and is assumed to be different from zero), whereas $\kappa^{*}$ is a nonhomogeneous linear function of the curvature $\boldsymbol{\theta}_{2}$, of the type $\kappa^{*}=\kappa_{0}^{*}+\boldsymbol{L} \boldsymbol{\theta}_{2}$, with the coefficients of $\boldsymbol{L}$ not all zero by hypothesis. Therefore, close to $\mathcal{S}$, eigenvalue $\lambda=\varepsilon \lambda_{1}+\mathcal{O}\left(\varepsilon^{2}\right)$ with $\lambda_{1}=\lambda_{1}\left(\boldsymbol{\theta}_{2}\right)$.

To discuss Eq. (18), it is convenient to determine first two codimension- 1 manifolds, both tangent to $\mathcal{S}$ at $\boldsymbol{\theta}=\mathbf{0}$, on which one critical eigenvalue is zero, for example, $\mathcal{D}$ (divergence boundary), or the two critical eigenvalues coincide, for example, $\mathcal{N}$ (nilpotent system family). To evaluate $\mathcal{D}$ and $\mathcal{N}$, coefficients $\kappa^{*}=0$ and $\xi^{2} / 4$, respectively, are required, from which linear equations in the $\boldsymbol{\theta}_{2}$ unknown are obtained. The two manifolds divide the space into three parts in which the eigenvalues are 1) real and opposite in sign, 2) real with the same sign, or 3) complex conjugate. The scenario of Fig. 1 consequentlyalters, as shown in Fig. 2, according to the value of $\kappa_{0}^{*}$.

The analysis of the neighborhood of $\mathcal{S}$, therefore, permits a more accurate description of the divergence boundary and, most important, the discovery of the existence of a region of the parameter space in which double divergence occurs. Because this region is bounded by two tangent manifolds, it cannot be identified by a first-order perturbation analysis, in which the parameters are varied proportionally. Moreover, the analysis highlights the existence of a family $\mathcal{N}$ of defectivesystems, to which the double-zeroeigenvalue system $\boldsymbol{A}_{0}$ belongs.

Previous results have shown that, in the generic case, the doublezero eigenvalue arises at the intersection of a $\mathcal{D}$ manifold and a $\mathcal{H}$ manifold. The scenario is much more complex in the nongeneric
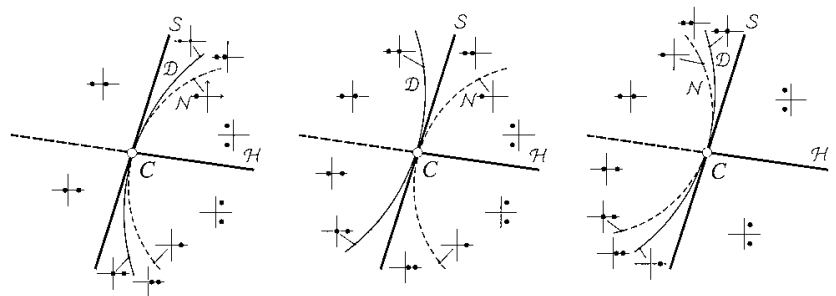

a) $\kappa_{0}^{*}<0$

b) $0<\kappa_{0}^{*}<\xi^{2} / 4$

c) $\kappa_{0}^{*}>\xi^{2} / 4$

Fig. 2 Stability diagram in the generic case: refined analysis. case in which all the coefficients of $\boldsymbol{L}$ vanish. A brief outline of the problem is given here (see Fig. 3, where a bidimensional case is represented).

In the nongeneric case, Eqs. (18) and (19) hold in whole space. Therefore, $\lambda=\varepsilon \lambda_{1}+\mathcal{O}\left(\varepsilon^{2}\right)$, with $\lambda_{1}$ still given by Eq. (18). However, coefficients $\xi$ and $\kappa^{*}$ are now homogeneous functions of the $\boldsymbol{\theta}_{1}$ unknown, linear and quadratic, respectively; on the contrary, $\boldsymbol{\theta}_{2}$ does not appear at this order because $\boldsymbol{L} \boldsymbol{\theta}_{2}=0$ by hypothesis. By requiring $\xi=0$, we have one value of $\boldsymbol{\theta}_{1}$ drawn; if $\kappa^{*}$ correspondingly assumes positive values, an $\mathcal{H}$ manifold is found (cases shown in Figs. 3a, 3b, and 3e), but if $\kappa^{*}$ is negative, no Hopf boundary exists (Figs. $3 \mathrm{c}$ and $3 \mathrm{~d}$ ). Note that in the nongeneric case $\mathcal{H}$ is a subspace, different from the generic case in which it is a half-subspace.Then, by requesting $\kappa^{*}=0$, we obtain zero or two real values of $\boldsymbol{\theta}_{1}$, from which zero or two $\mathcal{D}$ manifolds are found (Figs. $3 \mathrm{a}$ and $3 \mathrm{~b}$ or $3 \mathrm{c}-3 \mathrm{e}$, respectively). Similarly, if $\kappa^{*}=\xi^{2} / 4$, is requested, zero or two real $\mathcal{N}$ manifolds exist (Figs. $3 \mathrm{a}$ and $3 \mathrm{c}$ or $3 \mathrm{~b}, 3 \mathrm{~d}$, and $3 \mathrm{e}$, respectively). Therefore, by the exclusion of the case $\kappa^{*}<0 \forall \boldsymbol{\theta}$ for which $\boldsymbol{\theta}=\mathbf{0}$ is always unstable, the double-zero eigenvalue either occurs at the intersection of two divergence boundaries (as predicted in Sec. II in the singular case $I_{n, \boldsymbol{\theta}}=\mathbf{0}$, Figs. $3 \mathrm{c}$ and 3d) or simply lies on a Hopf boundary (Figs. 3a and 3b), as well as at the intersection of the three manifolds (Fig. 3e). Figure 3 is exhaustive of all possible cases, if coalescence among the manifolds is excluded.

\section{Equivalent Single-Degree-of-Freedom System}

The preceding analysis has shown that a complete description of the character of the critical eigenvalues around the origin of the parameter space requires using two different asymptotic expansions, each valid only for nonsingular or singular perturbations. However, the two expansions do not permit analysis of nearly singular perturbations that occur when $\lambda_{1 / 2}= \pm\left(\boldsymbol{v}_{2}^{T} \boldsymbol{A}_{1} \boldsymbol{u}_{1}\right)^{1 / 2}$ is not exactly zero but is small of order $\varepsilon$. From a geometrical point of view this case occurs when points of the parameter space close to the singular subspace $\mathcal{S}$ are considered. If these points are reached from the origin by following straight paths, the nonsingular solution furnishes incorrect results because $\lambda_{1 / 2}$ is small along them. Therefore, the neighborhood of $\mathcal{S}$ must be spanned by parabolas tangent to $\mathcal{S}$ itself, and the singular solution used.

Because of these drawbacks, it would be desiderable to obtain a unique expression $\lambda=\lambda(\boldsymbol{\theta})$ for the critical eigenvalues, uniformly valid in the whole space; moreover, it would be convenient to span the space through straight lines instead of curves. To this end, note that the stability diagrams of Fig. 2 are topologically equivalent to the stability diagram of the equilibrium position of a damped single-degree-of-freedom (DOF) elastic system. Its eigenvalues are

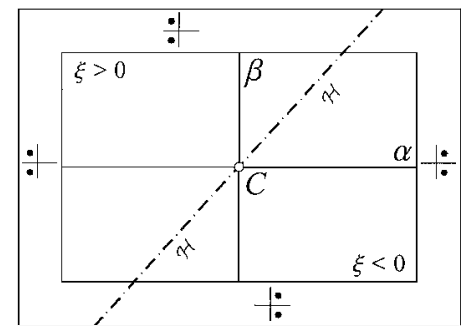

a) $\kappa^{*}>\mathbf{0}, \Delta<0$

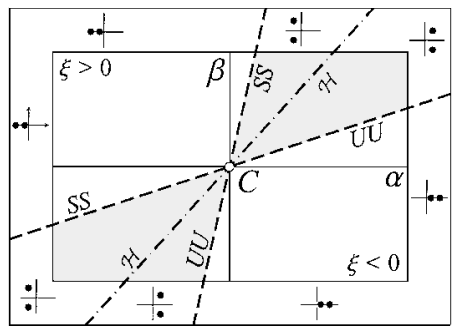

b) $\kappa^{*}>\mathbf{0}, \Delta \gtrless \mathbf{0}$

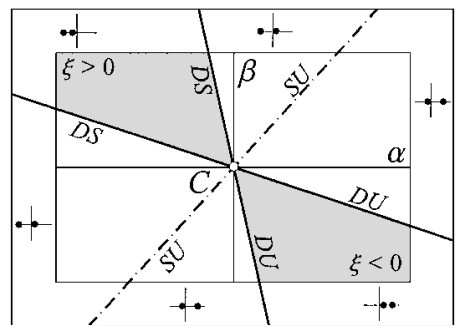

c) $\kappa^{*} \gtrless \mathbf{0}, \Delta>\mathbf{0}$

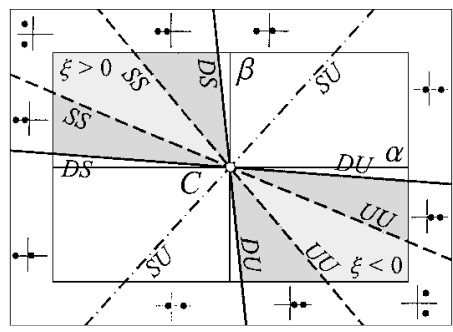

d) $\kappa^{*} \gtrless \mathbf{0}, \Delta \gtrless \mathbf{0}$

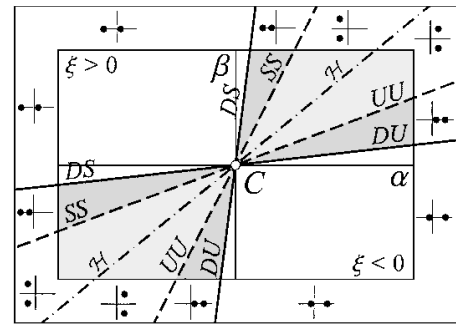

e) $\kappa^{*} \gtrless 0, \Delta \gtrless 0 ; \Delta:=\left(\xi^{2}-4 \kappa^{*}\right)^{\frac{1}{2}}$

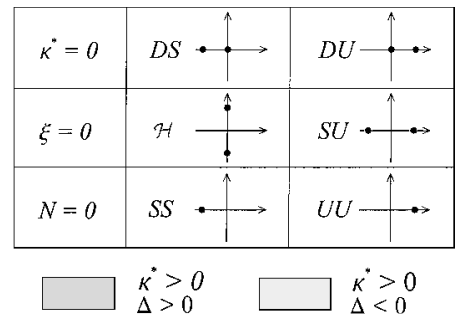

Fig. 3 Stability diagram in the nongeneric case: —_, divergence boundaries; - - - , nilpotent families $(\Delta=0)$; and - --, Hopf boundaries. 
governed by the characteristic equation

$$
\lambda^{2}+\xi \lambda+\kappa=0
$$

with $\xi$ the damping and $\kappa$ the stiffness coefficient. For the singleDOF system, $\kappa=0$ is the divergence boundary $\mathcal{D}, \xi=0$ the Hopf boundary $\mathcal{H}$, and $\kappa=\xi^{2} / 4$ the family of defectivesystems $\mathcal{N}$. Therefore, it is to be expected that a suitable definition of the coefficients $\xi$ and $\kappa$ exists in terms of the original parameters $\boldsymbol{\theta}$, such that Eq. (20) describes the behavior of the $n$-dimensional system $\boldsymbol{A}(\boldsymbol{\theta})$ around the double-zero eigenvalue. In other words, the task is to find an equivalent small-dimensional system, able to capture the qualitative behavior of the larger dimensional system, similar to that pursued by means of the center manifold procedure in the nonlinear stability analysis.

Straight lines through the origin of the parameter space are considered, with equations $\boldsymbol{\theta}=\varepsilon \boldsymbol{\theta}$. Correspondingly, the Jacobian matrix along them reads $\boldsymbol{A}=\boldsymbol{A}_{0}+\varepsilon \boldsymbol{A}_{1}+\varepsilon^{2} \boldsymbol{A}_{2}+\cdots$, with $\boldsymbol{A}_{1}=\boldsymbol{A}_{\boldsymbol{\theta}}^{0} \boldsymbol{\theta}$ and $A_{2}=A_{\theta \theta}^{0} \theta^{2} / 2$. Then, let us assume the whole spectrum of the defective matrix $\boldsymbol{A}_{0}$ is known. Let $\left\{\lambda_{0}, \lambda_{0} ; \lambda_{3}, \ldots, \lambda_{k}, \ldots\right.$, $\left.\lambda_{n}\right\}$ where $\lambda_{0}=0$ are the eigenvalues of $\boldsymbol{A}_{0}$ and $\boldsymbol{U}=\left\{\boldsymbol{u}_{1}, \boldsymbol{u}_{2}\right.$; $\left.\boldsymbol{u}_{3}, \ldots, \boldsymbol{u}_{k}, \ldots, \boldsymbol{u}_{n}\right\}$ and $\boldsymbol{V}=\boldsymbol{U}^{-1}=\left\{\boldsymbol{v}_{1}, \boldsymbol{v}_{2} ; \boldsymbol{v}_{3}, \ldots, \boldsymbol{v}_{k}, \ldots, \boldsymbol{v}_{n}\right\}$ and the matrices of the right and left eigenvectors, respectively, and $\boldsymbol{u}_{2}$ and $v_{1}$ are generalized eigenvectors. In the base of the eigenvectors, the eigenvalue problem (1) reads

$$
\left(\boldsymbol{J}_{0}+\varepsilon \boldsymbol{J}_{1}+\varepsilon^{2} \boldsymbol{J}_{2}\right) z-\lambda \boldsymbol{z}=\mathbf{0}
$$

where $\boldsymbol{J}_{0}=\boldsymbol{V}^{H} \boldsymbol{A}_{0} \boldsymbol{U}$ is the Jordan form of $\boldsymbol{A}_{0}, \boldsymbol{J}_{i}=\boldsymbol{V}^{H} \boldsymbol{A}_{i} \boldsymbol{U}$ $(i=1,2)$ are the first-and second-orderperturbationmatrices in the new base, and $z=V \boldsymbol{w}$. The characteristic equation of the eigenvalue problem (21) is

$\left\|\begin{array}{cccccc}\epsilon_{11}-\lambda & 1+\epsilon_{12} & \epsilon_{13} & \epsilon_{14} & \cdots & \epsilon_{1 n} \\ \epsilon_{21} & \epsilon_{22}-\lambda & \epsilon_{23} & \epsilon_{24} & \cdots & \epsilon_{2 n} \\ \epsilon_{31} & \epsilon_{32} & \lambda_{3}-\lambda+\epsilon_{33} & \epsilon_{34} & \cdots & \epsilon_{3 n} \\ \epsilon_{41} & \epsilon_{42} & \epsilon_{43} & \lambda_{4}-\lambda+\epsilon_{44} & \cdots & \epsilon_{4 n} \\ \cdots & \cdots & \cdots & \cdots & \cdots & \cdots \\ \epsilon_{n 1} & \epsilon_{n 2} & \epsilon_{n 3} & \epsilon_{n 4} & \cdots & \lambda_{n}-\lambda+\epsilon_{n n}\end{array}\right\|$

$$
=0
$$

where

$$
\epsilon_{i j}=\varepsilon v_{i}^{T} \boldsymbol{A}_{1} \boldsymbol{u}_{j}+\varepsilon^{2} \boldsymbol{v}_{i}^{T} \boldsymbol{A}_{2} \boldsymbol{u}_{j}
$$

are quantities of order $\varepsilon$, accounting for perturbations. To study Eq. (22), the dominant minors $\Delta_{k}$ of order $k=2,3, \ldots, n$ are successively evaluated asymptotically. The order-two minor is

$$
\Delta_{2}=\lambda^{2}-\lambda\left(\epsilon_{11}+\epsilon_{22}\right)+\epsilon_{11} \epsilon_{22}-\epsilon_{21}\left(1+\epsilon_{12}\right)
$$

If we let $\Delta_{2}=0$, the lower-order approximation to $\lambda$ is found, in which the contribution of the passive eigenvalues is ignored. If the key term $\epsilon_{21}=\mathcal{O}(\varepsilon)$ (nonsingularcase), then $\lambda=\mathcal{O}\left(\varepsilon^{1 / 2}\right)$, whereas if $\epsilon_{21}=\mathcal{O}\left(\varepsilon^{2}\right)$ (singularcase), then $\lambda=\mathcal{O}(\varepsilon)$. If we considerhigherorder minors, it is found that

$$
\begin{aligned}
\Delta_{3}= & \left(\lambda_{3}-\lambda+\epsilon_{33}\right) \Delta_{2}+\epsilon_{31} \epsilon_{23}+\mathcal{O}\left(\varepsilon^{3}, \varepsilon^{2} \lambda\right) \\
\Delta_{4}= & \left(\lambda_{4}-\lambda+\epsilon_{44}\right) \Delta_{3}+\left(\lambda_{3}-\lambda+\epsilon_{33}\right) \epsilon_{41} \epsilon_{24}+\mathcal{O}\left(\varepsilon^{3}, \varepsilon^{2} \lambda\right) \\
\Delta_{5}= & \left(\lambda_{5}-\lambda+\epsilon_{55}\right) \Delta_{4}+\left(\lambda_{3}-\lambda+\epsilon_{33}\right)\left(\lambda_{4}-\lambda+\epsilon_{44}\right) \epsilon_{51} \epsilon_{25} \\
& +\mathcal{O}\left(\varepsilon^{3}, \varepsilon^{2} \lambda\right) \\
& \ldots \ldots \ldots \ldots \ldots \\
\Delta_{n}= & \left(\lambda_{n}-\lambda+\epsilon_{n n}\right) \Delta_{n-1}+\prod_{k}^{n-1}\left(\lambda_{k}-\lambda+\epsilon_{k k}\right) \epsilon_{k 1} \epsilon_{2 k} \\
+ & \mathcal{O}\left(\varepsilon^{3}, \varepsilon^{2} \lambda\right)
\end{aligned}
$$

where terms of order higher than $\varepsilon^{2}$ have been neglected. By the use of Eqs. (25) in sequence, Eqs. (22) finally reads

$$
\begin{gathered}
\lambda^{2}-\lambda\left(\epsilon_{11}+\epsilon_{22}\right)+\epsilon_{11} \epsilon_{22}-\epsilon_{21}\left(1+\epsilon_{12}\right) \\
+\sum_{k}^{n} \frac{1}{\lambda_{k}} \epsilon_{k 1} \epsilon_{2 k}+\mathcal{O}\left(\varepsilon^{3}, \varepsilon^{2} \lambda\right)=0
\end{gathered}
$$

The former is the single-DOF equation (20) sought, from which the equivalent damping and stiffness coefficients are

$$
\begin{gathered}
\xi:=-\left(v_{2}^{T} A_{1} u_{2}+v_{1}^{T} A_{1} u_{1}\right) \\
\kappa:=-v_{2}^{T} A_{1} u_{1}\left(1+v_{1}^{T} A_{1} u_{2}\right)-v_{2}^{T} A_{2} u_{1}-v_{2}^{T} A_{1} \hat{w}_{1}
\end{gathered}
$$

In Eqs. (27) terms up to $\varepsilon^{2}$ order have been retained, consistent with the error contained in Eq. (26); moreover, use has been made of Eqs. (A4b) in the Appendix and the parameter $\varepsilon$ has been reabsorbed, that is, $\boldsymbol{A}_{1}=\boldsymbol{A}_{\boldsymbol{\theta}}^{0} \boldsymbol{\theta}$ and $\boldsymbol{A}_{2}=\boldsymbol{A}_{\boldsymbol{\theta} \boldsymbol{\theta}}^{0} \boldsymbol{\theta}^{2} / 2$ should be read. Note that Eq. (26) differs from Eq. (24) in the presence of some extra terms accounting for the influence of the passive eigenvalues. Moreover, Eq. (27a) coincides with Eq. (19a), whereas Eq. (27b) contains more terms than Eq. (19b).

By solving the second-degree equation, we have

$$
\lambda=-\xi / 2 \pm \sqrt{\xi^{2} / 4-\kappa}
$$

To discuss the solution, the nonsingular and singular case are considered, and the relevant asymptotic solutions obtained earlier are compared with Eq. (28).

1) If $\boldsymbol{v}_{2}^{T} \boldsymbol{A}_{1} \boldsymbol{u}_{1} \neq 0$ (and it is of order-1, generic case), then $\kappa \simeq$ $-\boldsymbol{v}_{2}^{T} \boldsymbol{A}_{1} \boldsymbol{u}_{1}$. Because $\kappa$ and $\xi$ are of the same order, the square root in Eq. (28) can be expanded around $\xi=0$, so that

$$
\lambda= \pm \sqrt{-\kappa}-\xi / 2
$$

Equation (29) coincides with Eq. (15), so that Eq. (28) furnishes asymptotically the nonsingular solution. [However, Eq. (28) contains terms of higher order with respect to Eq. (29) that are not consistent with the order of Eq. (26); these terms may or may not improve the accuracy of solution (29). A discussion on a similar problem can be found in Ref. 14.] Note that, in the nonsingular case, the passive eigenvalues do not affect the two-term expansion of $\lambda$ that could, therefore, be obtained by restricting the analysis to the perturbations of the Jordan block, that is, from Eq. (24). However, the passive eigenvalues appear at the $\varepsilon^{2}$ order in Eq. (26) and, therefore, enter in the solution at the $\varepsilon^{3 / 2}$ order, in accordance with the perturbation scheme of Eq. (10).

2) If $\boldsymbol{v}_{2}^{T} \boldsymbol{A}_{1} \boldsymbol{u}_{1}=0$ (singularcase), then $\kappa=\kappa^{*}$ as given in Eq. (19b); moreover, $\kappa=\mathcal{O}\left(\xi^{2}\right)$ so that the square root in Eq. (28) cannot be expanded any more. Therefore, the lower approximation to $\lambda$ is given by Eq. (28) itself, which coincides with the solution to Eq. (18) obtained earlier. Consequently, even at the lower-order approximation, the critical eigenvalues are affected by the passive eigenvalues.

To sum up, the equivalent single-DOF system equation gives the same asymptotic results as the method in Sec. III. In principle, it requires the computation of the whole spectrum $\boldsymbol{A}_{0}$, whereas the former calls only for the solution of linear algebraic equations. However, the use of Eq. (A4b) renders knowledge of the spectrum of $\boldsymbol{A}_{0}$ unnecessary, so that the two procedures require the same computational effort. Moreover, the present method makes it possible to analyze the nearly singular case $\boldsymbol{v}_{2}^{T} \boldsymbol{A}_{1} \boldsymbol{u}_{1} \approx 0$ that cannot be studied by the former by using straight paths. In fact, this term should be shifted at a higher order in the perturbation procedure; however, the operation cannot be performed using the method of Sec. III because the term does not appear explicitly in the perturbation equations. In contrast, in the present method, Eq. (28) also holds in the nearly singular case, in analogy with Eq. (5); in this case the stiffness coefficient is given by the sum of two terms of the same order of magnitude, $\kappa=\boldsymbol{v}_{2}^{T} \boldsymbol{A}_{1} \boldsymbol{u}_{1}+\kappa^{*}$.

In conclusion, Eq. (28) furnishes asymptotic solutions valid in all singular, nonsingular, and nearly singular cases; therefore, it allows the description of the transition across the singularity. Moreover, it holds even in the case in which all of the coefficients of matrix $\boldsymbol{L}$ vanish, for which $\kappa=\kappa^{*}$ in the whole space.

Equation (28) also allows discussion of the geometrical meaning of the unfolding parameters of the Takens-Bogdanova bifurcation. The relevant bifurcation equation, in Bogdanova-Arnold form (see Refs. 9 and 10) reads as the equation of motion of a nonlinear single-DOF system, in which the unfolding parameters are just the 


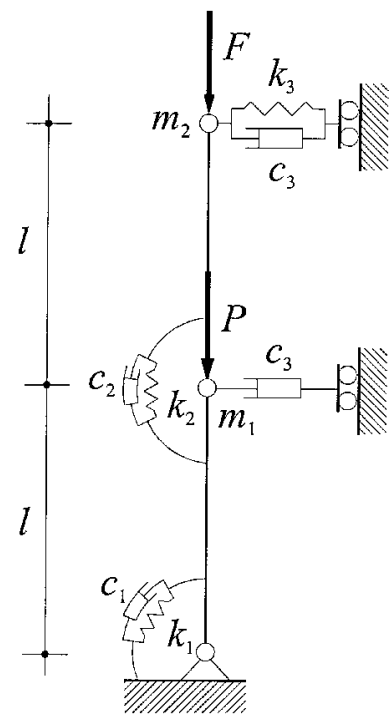

a) System parameters

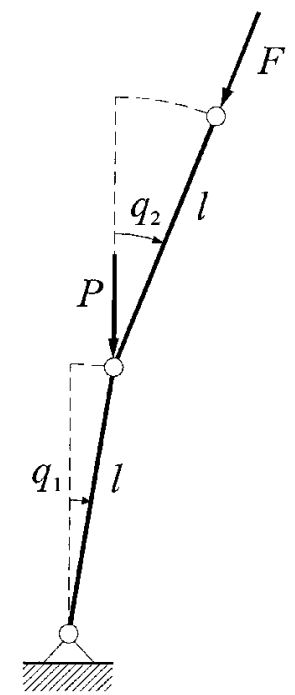

b) Lagrangian parameters
Fig. 4 Double pendulum system.

linear damping and stiffness coefficients. Therefore, Eqs. (27) supply explicit expressions for the unfolding parameters that were not available in the literature for general systems. For a two-parameter system $\boldsymbol{\theta}=\{\alpha, \beta\}$, varying $\xi$ and $\kappa$, Eq. (27a) defines, on the $(\alpha, \beta)$ plane, a family of straight lines parallel to the Hopf boundary $\xi=0$. Similarly, Eq. (27b) defines a family of parabolas obtained by translating the divergence boundary $\kappa=0$. If the quadratic terms in the parameters are neglected in Eq. (27b), the curves become straight lines parallel to the tangent at the divergence boundary at the critical point. It is concluded that the unfolding parameters $\xi$ and $\kappa$ are oblique coordinatesin the $(\alpha, \beta)$ plane, parallel to the critical boundaries at the criticality. The surprising finding in Refs. 11 and 12 is, therefore, not confirmed here.

\section{Illustrative Example}

The spectral properties of the structure shown in Fig. 4a are studied as an example. The structure consists of a double pendulum with lumped masses $m_{i}(i=1,2)$ and viscoelastic hinges of constants $c_{i}$ and $k_{i}$, elastically supported by a spring of constant $k_{3}$, subjected to a follower force $F$ and a dead load $P$. The external damping is assumed proportional to the speed of the masses through the coefficient $c_{3}$. Furthermore, $k_{2}=k_{1}, c_{2}=c_{1}, m_{1}=2 m$, and $m_{2}=m$ are taken, and the following dimensionless quantities are introduced:

$$
\begin{gathered}
a=k_{3} / m \omega^{2}, \quad b=F / m l \omega^{2}, \quad c=c_{1} / m l^{2} \omega \\
d=c_{3} l^{2} / c_{1}, \quad k=k_{1} / m l^{2} \omega^{2}, \quad p=P / m l \omega^{2}, \quad \tau=\omega t
\end{gathered}
$$

where $\omega$ is a scaling factor with the dimensions of a frequency. By choosing the rotations in Fig. $4 \mathrm{~b}$ as the Lagrangian coordinates, the dimensionless state vector $\boldsymbol{x} \in \mathbb{R}^{4}$ is $\boldsymbol{x}^{T}=\left(x_{1}, x_{2}, x_{3}, x_{4}\right)=$ $\left(q_{1}, \dot{q}_{1}, q_{2}, \dot{q}_{2}\right)$, where the dot denotes differentiation with respect to the time $\tau$. The linearized equations of motion around the trivial equilibrium position $\boldsymbol{x}=\mathbf{0}$ are found to be

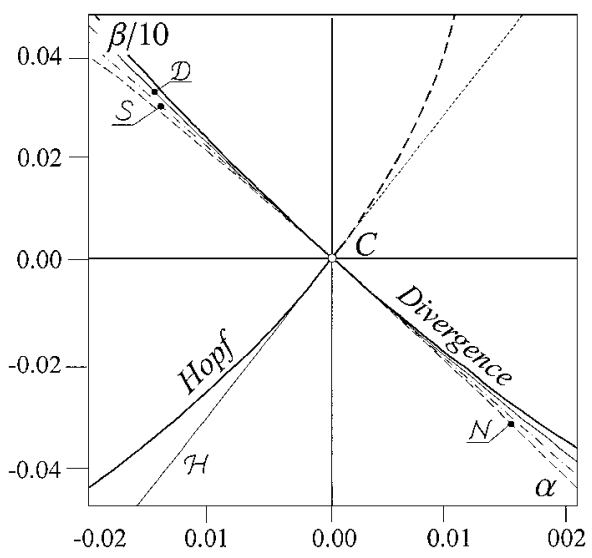

Fig. 5 Stability boundaries in the $(\alpha, \beta)$ parameter plane: - - exact solution; - --, nonsingular expansion [Eq. (15)]; and - - uniformly valid solution [Eq. (20)].

which defines the Jacobian matrix $\boldsymbol{A}$. The parameters $c, d, k$, and $p$ are taken as auxiliary parameters, and their values are fixed at $c=1.5, d=0.5, k=1$, and $p=0$, so that the system coincides with that analyzed in Ref. 12. By the variation of the control parameters $a$ and $b$ and the numerical solution of the eigenvalue problem associated with Eq. (31), a critical point $C$ of coordinates $\left(a_{c}, b_{c}\right)=(0.150,5.827)$ is found on the $(a, b)$ plane at the intersection of a Hopf and a divergence variety. The exact stability boundaries around $C$ are shown as heavy lines in Fig. 5, where the incremental parameters $\alpha:=a-a_{c}$ and $\beta:=b-b_{c}$ have been used. The perturbation analysis shown earlier was then applied. First, the right and left eigenvectors $\boldsymbol{u}_{1}, \boldsymbol{u}_{2}, \boldsymbol{v}_{1}$, and $\boldsymbol{v}_{2}$ were computed at $\alpha=\beta=0$; second, the perturbation matrices $\boldsymbol{A}_{1}$ and $\boldsymbol{A}_{2}$ and the solution $\hat{\boldsymbol{w}}_{1}$ to Eqs. (12) were evaluated as functions of the control parameters $\boldsymbol{\theta}=\{\alpha, \beta\}$; third, the coefficients $\xi, \kappa$, and $\kappa^{*}$ in Eqs. (19) and (27) were determined. If the nonsingular expansion Eq. (15) is applied, the tangents $\mathcal{S}$ and $\mathcal{H}$ to the exact boundaries are found (see Fig. 5); if Eq. (18) (valid only near $\mathcal{S}$ ) or Eq. (20) (valid in the whole plane) are applied instead, practically indistinguishable curves $\mathcal{D}$ and $\mathcal{N}$ are obtained. Thus, a slightly more accurate description of the divergence boundary is drawn, and a narrow region of real eigenvalues with the same sign, like that in Fig. 2b, is found.

To describe the behavior of the critical eigenvalues in the neighborhood of the origin of the $(\alpha, \beta)$ plane, it is convenientto introduce polarcoordinates $(\rho, \theta)$. By fixing $\rho=0.025$ and varying $\theta$ between 0 and $2 \pi$, the real and imaginary parts of the eigenvalues vary as shown in Figs. 6a and 6b by heavy lines; Figs. $6 \mathrm{c}$ and $6 \mathrm{~d}$ are closeups of Fig. 6a. Points $N_{1}, N_{2}, B_{1}$, and $B_{2}$ are bifurcation points for the eigenvalue locus at which the system becomes defective. However, whereas $N_{1}$ and $N_{2}$ arise from the interaction between the two critical eigenvalues, $B_{1}$ and $B_{2}$ involve the passive eigenvalues. At points $D_{1}$ and $D_{2}$, divergence takes place. Within $N$ points and $D$ points, two real eigenvalueshaving the same sign exist, according to the stability diagram in Figs. 5 and 2b. When a sensitivity analysis is performed, the approximate loci in Fig. 6 are obtained. It is seen that perturbation analysis does not describe the bifurcations $B_{1}$ and $B_{2}$ because no interactions among active and passive eigenvalues were taken into account. Except for these points, the approximation of the nonsingular solution is quite good. However, the closeups in Figs. $6 \mathrm{c}$ and $6 \mathrm{~d}$ reveal the existence of a local error around the $N$ points, which is due to the occurrence of a singularity on the $\mathcal{S}$ variety, also indicated in the Figs. 6 . In these zones, use should be made of Eq. (18) that, however, requires the expression of $\alpha$ and $\beta$

$$
\left\{\begin{array}{l}
\dot{x}_{1} \\
\dot{x}_{2} \\
\dot{x}_{3} \\
\dot{x}_{4}
\end{array}\right\}=\left[\begin{array}{cccc}
0 & 1 & 0 & 0 \\
(b-3 k-p) / 2 & -c(3+d) / 2 & (2 k-b) / 2 & c \\
0 & 0 & 0 & 1 \\
(5 k-b+p-2 a) / 2 & c(5-d) / 2 & (b-4 k-2 a) / 2 & -c(2+d)
\end{array}\right]\left\{\begin{array}{l}
x_{1} \\
x_{2} \\
x_{3} \\
x_{4}
\end{array}\right\}
$$




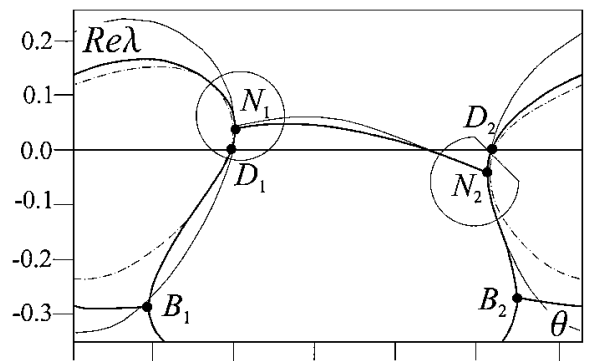

a) Real part

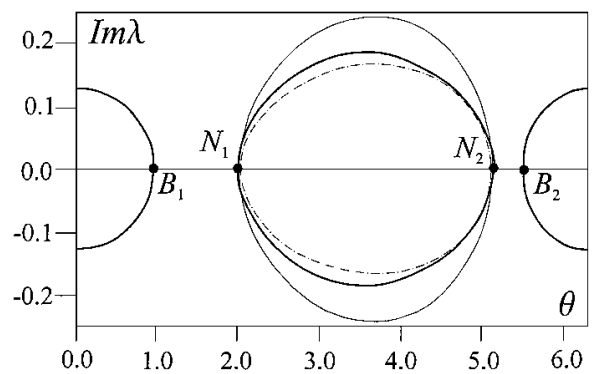

b) Imaginary part

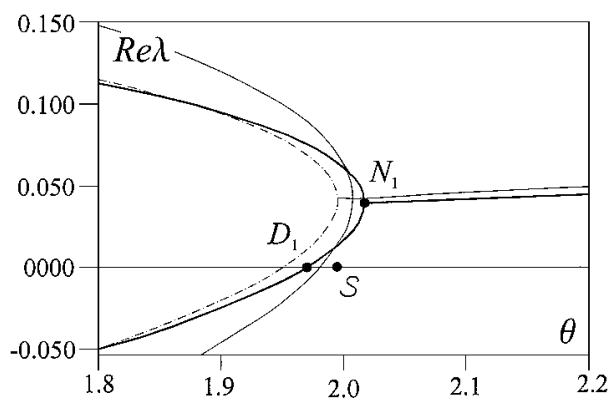

c) Closeup around point $N_{1}$

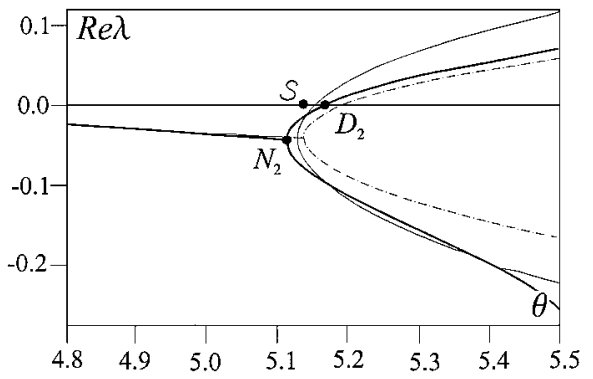

d) Closeup around point $N_{2}$

Fig. 6 Eigenvalues vs angle $\theta$ when $\rho=0.025$; lines as in Fig. 5 .
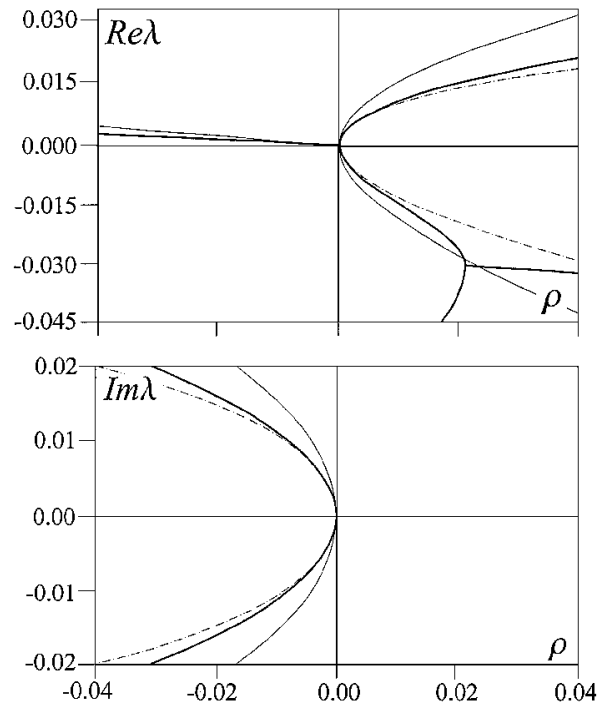

a) $\theta=\pi / 4 \mathrm{rad}$
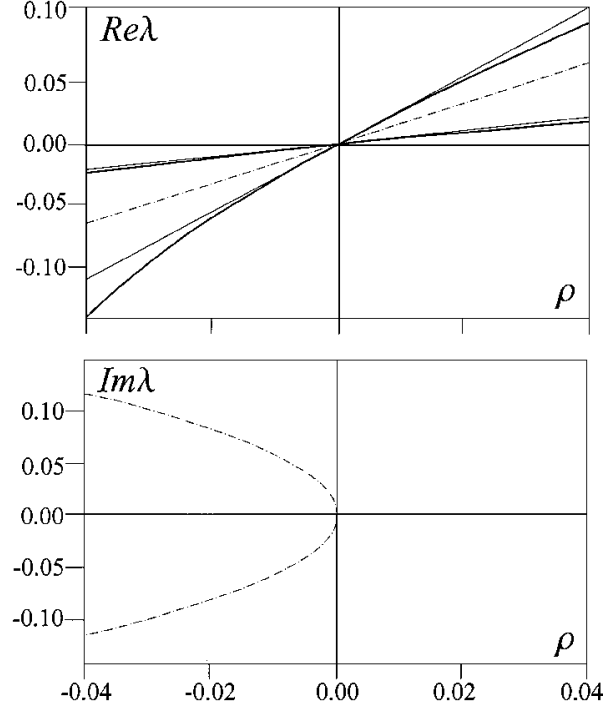

b) $\theta=1.995 \mathrm{rad}$

Fig. 7 Critical eigenvalues vs radius $\rho$ along the lines; lines as in Fig. 5.

(and consequently $\rho$ and $\theta$ ) in parametric form to describe parabolas tangent to $\mathcal{S}$. Therefore, it is much more convenient to use the uniformly valid Eq. (20) that appears to describe the eigenvalues around the singularity better than the nonsingular expansion; moreover, its approximation is satisfactory in the whole range of $\theta$.

The degree of accuracy of the approximation emerges more clearly from Figs. $7 \mathrm{a}$ and $7 \mathrm{~b}$, where the critical eigenvalues have been plotted as functions of $\rho$ for selected values of $\theta$. In Fig. 7a a generic value $\theta=\pi / 4 \mathrm{rad}$ is considered, whereas in Fig. 7b the special value $\theta=1.995 \mathrm{rad}$ corresponding to the singular variety $\mathcal{S}$ is taken into account. The result is that, far from $\mathcal{S}$ [where the eigenvaluesapproximately vary with $\rho^{1 / 2}$ (Fig. 7a)], the nonsingular and uniformly valid solutions both furnish a good approximation of the exact eigenvalues. In contrast, on $\mathcal{S}$ [where the eigenvalues vary almost proportionally to $\rho$ (Fig. 7b)], only the latter solution gives good results whereas the former solution is wrong.

The perturbation analysis developed also makes it possible to investigate the sensitivities of the critical point and of the stability boundaries, that is, the sensitivity of the whole stability diagram, to changes in the auxiliary parameters. Here the influence of the conservative load parameter $p$ is analyzed, while parameters $c, d$,

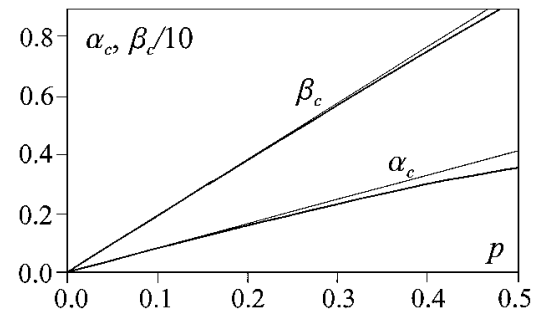

Fig. 8 Critical point coordinates $\alpha_{c}$ and $\beta_{c}$ vs conservative load factor $p:-$, exact solution, and —, uniformly valid solution [Eq. (20)].

and $k$ are kept fixed. The vector $\boldsymbol{\theta}$ is extended to include $p$, that is, $\theta=\{\alpha, \beta, p\}$; then the analysis is repeated to obtain the exact eigenvalues and all of the perturbation quantities as functions of the three parameters. The results concerning the modification of the critical point are plotted in Fig. 8. The differences between the exact and the approximated solution obtained by means of the uniformly valid expression become appreciable for values of $p$ near to 0.15 . This is confirmed in Fig. 9 by the comparison of the exact boundaries 

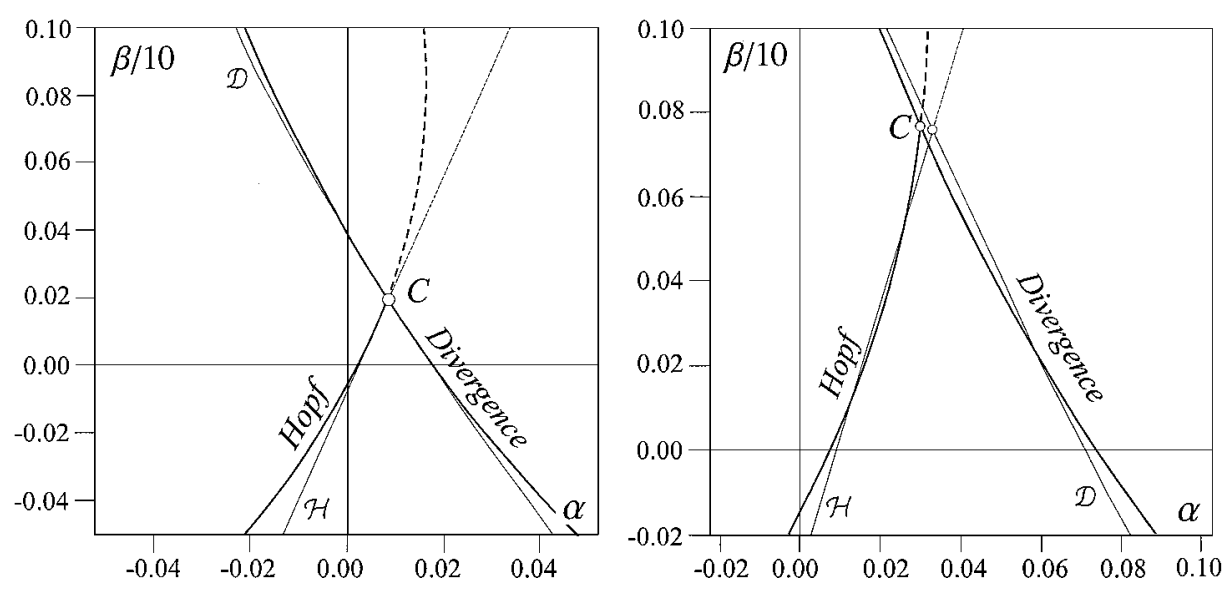

a) $p=0.1$

b) $p=0.4$

Fig. 9 Stability boundaries in the $(\alpha, \beta)$ parameter plane; lines as in Fig. 8.

of the Hopf divergence and the approximated curves. When $p=0.1$ (Fig. 3a), the approximate solution furnishes nearly exact tangents at the critical point; when $p=0.4$ (Fig. 9b) the approximationis still good, although an error on the tangents exists due to the approximate evaluation of the critical point.

Finally, the occurrence of the nongeneric case is studied. It is found that, if $c=1.5, d=5$, and $p=1$ are fixed and points are considered on the plane $2 a+b-5 k=1$, a singular case occurs at $\left(a_{c}, b_{c}\right)=\left(\frac{1}{3}, 2\right)$, for which $\boldsymbol{v}_{2}^{T} \boldsymbol{A}_{\alpha} \boldsymbol{u}_{1}=0$ and $\boldsymbol{v}_{2}^{T} \boldsymbol{A}_{\beta} \boldsymbol{u}_{1}=0$. In the problem, the case shown in Fig. $3 \mathrm{c}$ occurs, so that there is no Hopf bifurcation, but only a double divergence. As a particular case, the linearboundaries of Fig. $3 \mathrm{c}$ are found to coincide with the exact ones.

\section{Conclusions}

A sensitivity analysis of a double-zeroeigenvalue was performed and linear stability diagrams built up for a general multiparameter system. The following results were obtained.

1) At the critical point, due to the coalescence of two eigenvalues, the Jacobian matrix is defective because only a proper eigenvectoris associated with the double eigenvalue. Therefore, sensitivity analysis calls for the use of a series of fractional powers of the perturbation parameters. $^{6}$

2) In the generic case, the double-zero eigenvalue manifests itself at the intersection of a divergence manifold and a Hopf manifold in the parameter space; the classical Takens-Bogdanova bifurcation conditions are, therefore, verified. However, other bifurcation mechanisms exist in nongeneric cases, each leading to the double-zero bifurcation: the double divergence, the double divergence Hopf, and the degenerate Hopf. Moreover, even in the generic case, the subspace tangent to the divergence manifold is found to be a locus of singular systems for which sensitivities are of lower order.

3) A region exists in the parameter space in which the eigenvalues are both real and positive. It is bounded by the divergence manifold and a second manifold on which the eigenvalues are real, different from zero, and still coalescent. Therefore, this manifold is a locus of defective systems, to which the critical system belongs. To detect the double divergence region, perturbationsalong a parabola tangent to the singular subspace must be performed.

4) Different asymptotic approaches were discussed. In particular, a second-degree equation for the critical eigenvalue was found that is uniformly valid around the critical point and also keeps its validity in the nongeneric case. This equation is the characteristic equation of a damped single-DOFlinear system, whose damping and stiffness coefficients are suitably defined in terms of the perturbation of the defective matrix.

5) The equivalent damped oscillator equation also made it possible to clarify the geometricalmeaning of the unfolding parameters of the Takens-Bogdanova bifurcation. The damping and stiffness parameters are found to represent oblique coordinates exactly parallel to the tangents to the critical curves at the bifurcation point, similar to that happens for all codimension- 2 bifurcations. This result contrasts with some statements existing in the literature about this question. ${ }^{11,12}$

6) A mechanical two-DOF system was studied as an example. It was found that the non-singular perturbation expansion furnishes a good approximation almost everywhere in the parameter space, except in the neighborhood of the subspace tangent to the divergence manifold. In contrast, the uniformly valid solution accurately describes this neighborhood; furthermore it gives a good result outside the singular region. The (slight) loss of precision is attributed to inconsistent terms present in the solution. ${ }^{14}$

\section{Appendix: Solution to Equation (12) in the Base of the Right Eigenvectors of Matrix $A_{0}$}

The solution to Eq. (12) is expressed as

$$
\hat{\boldsymbol{w}}_{1}=\sum_{i}^{n} \alpha_{i} \boldsymbol{u}_{i}
$$

where $\left\{\boldsymbol{u}_{1}, \boldsymbol{u}_{2}\right\}$ are generalizedeigenvectorsassociated with the double eigenvalue $\lambda=0 ; \boldsymbol{u}_{k}(k=3, \ldots, n)$ are proper eigenvectors associated with the passive eigenvalues $\lambda_{k}(k=3, \ldots, n)$, assumed to be distinct; and $\alpha_{i}$ are unknown coefficients.

By substituting Eq. (A1) in Eq. (12a) and premultiplying it by the left eigenvectors $v_{j}^{H}$, we have

$$
\begin{array}{r}
\boldsymbol{v}_{j}^{H}\left(\alpha_{1} \boldsymbol{A}_{0} \boldsymbol{u}_{1}+\alpha_{2} \boldsymbol{A}_{0} \boldsymbol{u}_{2}+\sum_{k}^{n} \alpha_{k} \boldsymbol{A}_{0} \boldsymbol{u}_{k}\right)=\lambda_{\frac{1}{2}}^{2} \boldsymbol{v}_{j}^{H} \boldsymbol{u}_{2}-\boldsymbol{v}_{j}^{H} \boldsymbol{A}_{1} \boldsymbol{u}_{1} \\
(j=1,2, \ldots, n)
\end{array}
$$

By accounting for $\boldsymbol{A}_{0} \boldsymbol{u}_{1}=\mathbf{0}, \boldsymbol{A}_{0} \boldsymbol{u}_{2}=\boldsymbol{u}_{1}, \boldsymbol{A}_{0} \boldsymbol{u}_{k}=\lambda_{k} \boldsymbol{u}_{k}(k=3$, $\ldots, n)$, and for Eq. (11), we find that $\alpha_{2}=-\boldsymbol{v}_{1}^{T} \boldsymbol{A}_{1} \boldsymbol{u}_{1}, \alpha_{k}=$ $-\boldsymbol{v}_{k}^{T} \boldsymbol{A}_{1} \boldsymbol{u}_{1} / \lambda_{k}, \quad(k=3, \ldots, n)$, whereas $\alpha_{1}$ remains undetermined. By the enforcement of the orthogonality condition (12b), $\alpha_{1}=0$ follows, so that

$$
\hat{\boldsymbol{w}}_{1}=-\left(\boldsymbol{v}_{1}^{T} \boldsymbol{A}_{1} \boldsymbol{u}_{1}\right) \boldsymbol{u}_{2}-\sum_{k}^{n} \frac{\boldsymbol{v}_{k}^{H} \boldsymbol{A}_{1} \boldsymbol{u}_{1}}{\lambda_{k}} \boldsymbol{u}_{k}
$$

From Eq. (34) it follows that terms in Eqs. (13) and (19b) involving $\hat{\boldsymbol{w}}_{1}$ can be written as

$$
\begin{gathered}
\boldsymbol{v}_{2}^{T} \hat{\boldsymbol{w}}_{1}=-\boldsymbol{v}_{1}^{T} \boldsymbol{A}_{1} \boldsymbol{u}_{1} \\
\boldsymbol{v}_{2}^{T} \boldsymbol{A}_{1} \hat{\boldsymbol{w}}_{1}=-\left(\boldsymbol{v}_{1}^{T} \boldsymbol{A}_{1} \boldsymbol{u}_{1}\right)\left(\boldsymbol{v}_{2}^{T} \boldsymbol{A}_{1} \boldsymbol{u}_{2}\right)-\sum_{k}^{n} \frac{1}{\lambda_{k}}\left(\boldsymbol{v}_{k}^{H} \boldsymbol{A}_{1} \boldsymbol{u}_{1}\right)\left(\boldsymbol{v}_{2}^{T} \boldsymbol{A}_{1} \boldsymbol{u}_{k}\right)
\end{gathered}
$$

\section{Acknowledgment}

Of the funding, $40 \%$ was supported by the Italian Ministry of the University and Scientific and Technological Research in 1997. 


\section{References}

${ }^{1}$ Brandon, J. A., Strategies for Structural Dynamic Modification, WileyInterscience, New York, 1990

${ }^{2}$ Mottershead, J. E., and Friswell, M. I., "Model Updating in Structural Dynamics: A Survey," Journal of Sound and Vibration, Vol. 162, No. 3, 1993, pp. 347-375.

${ }^{3}$ Hou, G. J. W., and Kenny, S. P., "Eigenvalue and Eigenvector Approximate Analysis for Repeated Eigenvalue Problems," AIAA Journal, Vol. 30, No. 9, 1992, pp. 2317-2324

${ }^{4}$ Zhang, D. W., and Wei, F., "Computation of Eigenvector Derivatives with Repeated Eigenvalues Using a Complete Modal Space," AIAA Journal, Vol. 33, No. 9, 1995, pp. 1749-1753.

${ }^{5}$ Zheng, S., Ni, W., and Wang, W., "Combined Method for Calculating Eigenvector with Repeated Eigenvalues," AIAA Journal, Vol. 36, No. 3, 1998, pp. 428-431.

${ }^{6}$ Luongo, A., "Eigensolutions Sensitivity for Nonsymmetric Matrices with Repeated Eigenvalues," AIAA Journal, Vol. 31, No. 7, 1993, pp. 13211328.

${ }^{7}$ Xu, T., Chen, S., and Liu, Z., "Perturbation Sensitivity of Generalized Modes of Defective Systems," Computers and Structures, Vol. 52, No. 2, 1994, pp. 179-185.
${ }^{8}$ Luongo, A., and Paolone, A., "Perturbation Methods for Bifurcation Analysis from Multiple Nonresonant Complex Eigenvalues," Nonlinear Dynamics, Vol. 14, No. 3, 1997, pp. 193-210.

${ }^{9}$ Guckenheimer, J., and Holmes, P. J., Nonlinear Oscillations, Dynamical Systems and Bifurcations of Vector Fields, Springer-Verlag, New York, 1983, pp. 364-376.

${ }^{10}$ Arnold, V. I., Geometrical Methods in the Theory of Ordinary Differential Equations, Springer-Verlag, New York, 1983, pp. 280-282 (Russian original, Moscow, 1977).

${ }^{11}$ Holmes, P. J., "Center Manifolds, Normal Forms and Bifurcations of Vector Fields with Application to Coupling Between Periodic and Steady Motions," Physica 2D, 1981, pp. 449-481.

${ }^{12}$ Troger, H., and Steindl, A., Nonlinear Stability and Bifurcation Theory, Springer-Verlag, Vienna, 1991, p. 259.

${ }^{13}$ Luongo, A., "Eigensolutions of Perturbed Nearly Defective Matrices," Journal of Sound and Vibration, Vol. 185, No. 3, 1995, pp. 377-395.

${ }^{14}$ Luongo, A., and Paolone, A., "On the Reconstitution Problem in the Multiple Time Scale Method," Nonlinear Dynamics, Vol. 19, No. 2, 1999. pp. 133-156.
A. Berman Associate Editor 